\title{
Inferior survival for patients with malignant peripheral nerve sheath tumors defined by aberrant TP53
}

\author{
Maren Høland $\mathbb{1}^{1,2} \cdot$ Matthias Kolberg $^{1} \cdot$ Stine Aske Danielsen ${ }^{1} \cdot$ Bodil Bjerkehagen $^{3,4} \cdot$ Ina A. Eilertsen ${ }^{1}$. \\ Merete Hektoen $^{1} \cdot$ Nils Mandahl $^{5} \cdot$ Eva van den Berg ${ }^{6} \cdot$ Sigbjørn Smeland ${ }^{2,7} \cdot$ Fredrik Mertens $\mathbb{D}^{5}$. \\ Kirsten Sundby Hall ${ }^{7}$ Piero Picci $\mathbb{1}^{8} \cdot$ Anita Sveen $^{1} \cdot$ Ragnhild A. Lothe ${ }^{1,2}$
}

Received: 2 January 2018 / Revised: 22 April 2018 / Accepted: 22 April 2018 / Published online: 26 June 2018

(c) United States \& Canadian Academy of Pathology 2018

\begin{abstract}
Malignant peripheral nerve sheath tumor is a rare and aggressive disease with poor treatment response, mainly affecting adolescents and young adults. Few molecular biomarkers are used in the management of this cancer type, and although TP53 is one of few recurrently mutated genes in malignant peripheral nerve sheath tumor, the mutation prevalence and the corresponding clinical value of the TP53 network remains unsettled. We present a multi-level molecular study focused on aberrations in the TP53 network in relation to patient outcome in a series of malignant peripheral nerve sheath tumors from 100 patients and 38 neurofibromas, including TP53 sequencing, high-resolution copy number analyses of TP53 and MDM2, and gene expression profiling. Point mutations in TP53 were accompanied by loss of heterozygosity, resulting in complete loss of protein function in $8.2 \%$ of the malignant peripheral nerve sheath tumors. Another $5.5 \%$ had MDM2 amplification. TP53 mutation and MDM2 amplification were mutually exclusive and patients with either type of aberration in their tumor had a worse prognosis, compared to those without (hazard ratio for 5-year disease-specific survival 3.5, 95\% confidence interval 1.78-6.98). Both aberrations had similar consequences on the gene expression level, as analyzed by a TP53associated gene signature, a property also shared with the copy number aberrations and/or loss of heterozygosity at the TP53 locus, suggesting a common "TP53-mutated phenotype" in as many as $60 \%$ of the tumors. This was a poor prognostic phenotype (hazard ratio $=4.1$, confidence interval:1.7-9.8), thus revealing a TP53-non-aberrant patient subgroup with a favorable outcome. The frequency of the "TP53-mutated phenotype" warrants explorative studies of stratified treatment strategies in malignant peripheral nerve sheath tumor.
\end{abstract}

\section{Introduction}

Malignant peripheral nerve sheath tumor is a rare and highly aggressive malignancy with a 5-year survival rate lower than

Electronic supplementary material The online version of this article (https://doi.org/10.1038/s41379-018-0074-y) contains supplementary material, which is available to authorized users.

Ragnhild A. Lothe

rlothe@rr-research.no

1 Department of Molecular Oncology, Institute for Cancer Research, Oslo University Hospital, Oslo, Norway

2 Institute for Clinical Medicine, University of Oslo, Oslo, Norway

3 Department of Oral Biology, University of Oslo, Oslo, Norway

4 Department of Pathology, Division of Laboratory Medicine, Oslo University Hospital, Oslo, Norway
$50 \%$ [1]. About half of the cases arise in individuals with neurofibromatosis type 1 , while the remaining tumors are sporadic. The neurofibromatosis type 1 syndrome, caused by an inherited or de novo mutation in the tumor suppressor gene $N F 1$, is one of the most common autosomal dominant disorders, with an incidence of one in 2500-3000 individuals [2]. The syndrome affects men and women equally, and the

5 Department of Clinical Genetics, University and Regional Laboratories, Lund University, Lund, Sweden

6 Department of Genetics, The University Medical Center Groningen, Groningen, The Netherlands

7 Department of Oncology, Division of Cancer Medicine, Oslo University Hospital, Oslo, Norway

8 Laboratory of Experimental Oncology, Istituto Ortopedico Rizzoli, Bologna, Italy 
Table 1 Literature survey of TP53 mutation analysis in malignant peripheral nerve sheath tumors

\begin{tabular}{|c|c|c|c|c|c|c|c|}
\hline Year & First author & Method & Sample type & $\begin{array}{l}\text { Exons } \\
\text { analyzed }\end{array}$ & $\begin{array}{l}\text { Number of } \\
\text { tumors mutated }^{\mathrm{e}}\end{array}$ & $\begin{array}{l}\text { Number of } \\
\text { tumors analyzed }^{\mathrm{e}}\end{array}$ & $\begin{array}{l}\% \text { with } \\
\text { mutation }^{\mathrm{f}}\end{array}$ \\
\hline 1989 & Nigro & PCR and ds & Biopsy/cell lined $^{\mathrm{d}}$ & 2 to 11 & 1 & 1 & 100 \\
\hline 1990 & Menon & PCR and bds & Fresh frozen & 5 to 8 & 1 & 7 & 14.3 \\
\hline 1993 & Boman & PCR-SSCP and ds & Fresh frozen & 2 to 11 & 0 & 1 & 0 \\
\hline 1993 & Lothe $^{\mathrm{a}}$ & PCR CDGE and ds & Fresh frozen & $5,7,8$ & 0 & 1 & \\
\hline 1994 & Flørenes & PCR-SSCP and ds & Fresh frozen & $5,7,8$ & 0 & 3 & 0 \\
\hline 1994 & Latres & PCR-SSCP and ds & Fresh frozen & 2 to 9 & 0 & 6 & 0 \\
\hline 1994 & Legius & PCR and solid phase ds & Fresh frozen/cell line ${ }^{\mathrm{d}}$ & 4 to 9 & 2 & 3 & 66.7 \\
\hline 1995 & Castresana & PCR-SSCP and ds & Fresh frozen & 5 to 8 & 1 & 9 & 11.1 \\
\hline 1995 & Lothe $^{\mathrm{a}}$ & PCR CDGE and ds & Fresh frozen & 5 to 8 & 0 & 7 & \\
\hline 1997/ & $\begin{array}{l}\text { Schneider- } \\
\text { Stock }{ }^{\mathrm{b}} /\end{array}$ & PCR-SSCP and ds & Fresh frozen & 5 to 8 & $4(3,1)$ & $18(10,12)$ & 22.2 \\
\hline 2002 & Mawrin $^{\text {b }}$ & & & & & & \\
\hline 1998 & Taubert & PCR-SSCP and ds & $\begin{array}{l}\text { Fresh frozen/formalin- } \\
\text { fixed }\end{array}$ & 4 to 9 & 0 & 28 & 0 \\
\hline 1999 & Rieske & PCR-SSCP and ds & Fresh frozen & 5 to 8 & 2 & 15 & 13.3 \\
\hline 2000 & Rasmussen & PCR-SSCP and ds & Not given & 4 to 9 & 0 & 5 & 0 \\
\hline 2000 & Sonobe & PCR and ds & Cell line ${ }^{\mathrm{d}}$ & 4 to 9 & 1 & 1 & 100 \\
\hline 2001 & Birindelli & PCR-SSCP and ds & Formalin-fixed & 5 to 8 & 7 & 27 & 25.9 \\
\hline 2001 & Lefevre & PCR and ds & Fresh frozen & 2 to 11 & 1 & 1 & 100 \\
\hline 2001 & Leroy & PCR-DGGE and ds & Fresh frozen & 5 to 8 & 4 & 6 & 66.7 \\
\hline 2001 & Lothe $^{\mathrm{a}}$ & $\begin{array}{l}\text { PCR-DG/GE/TGGE and } \\
\text { ds }\end{array}$ & Fresh frozen & 2 to 11 & 0 & 16 & \\
\hline 2001 & Watanabe & PCR-SSCP and ds & Formalin-fixed & 5 to 8 & 0 & 17 & 0 \\
\hline 2002 & Shin & PCR and ds & Formalin-fixed & 5 to 8 & 1 & 1 & 100 \\
\hline 2004 & Magrini & PCR and ds & Fresh frozen & 4 to 10 & 1 & 1 & 100 \\
\hline 2004 & Upadhyaya & PCR-SSCP and ds & Not given & 4 to 9 & 0 & 6 & 0 \\
\hline 2007 & $\mathrm{Chao}^{\mathrm{c}}$ & PCR and ds & Leukocytes & 4 to 10 & 1 & 1 & \\
\hline 2007 & Holtkamp & PCR-SSCP and ds & $\begin{array}{l}\text { Fresh frozen/formalin- } \\
\text { fixed }\end{array}$ & 1 to 11 & 4 & 36 & 11.1 \\
\hline 2008 & Upadhyaya & PCR and ds & Not given & 4 to 9 & 4 & 15 & 26.7 \\
\hline 2009 & Fauth & PCR and ds & Formalin-fixed & 2 to 11 & 1 & 2 & 50.0 \\
\hline 2010 & Verdijk & PCR and bds & Formalin-fixed & 4 to 9 & 17 & 72 & 23.6 \\
\hline 2011 & Beert & PCR and bds & Cell line ${ }^{\mathrm{d}}$ & 2 to 9 & 3 & 6 & 50.0 \\
\hline 2012 & Evans $^{c}$ & not given & Not given & Not given & 3 & 3 & \\
\hline 2014 & Lee & $\begin{array}{l}\text { WGS }(n=12) / \mathrm{TS}(n= \\
32)\end{array}$ & $\begin{array}{l}\text { Fresh frozen/formalin- } \\
\text { fixed }^{\mathrm{g}}\end{array}$ & Full & 11 & 44 & 25.0 \\
\hline 2014 & Zhang & $\begin{array}{l}\text { WGS }(n=4) / \operatorname{WES}(n= \\
\text { 4) }\end{array}$ & Fresh frozen & Full & 1 & 8 & 12.5 \\
\hline 2015 & Hirbe & WES/TS & $\begin{array}{l}\text { Fresh frozen/formalin- } \\
\text { fixed }\end{array}$ & Full & 3 & 16 & 18.8 \\
\hline 2015 & McPherson & WES & Fresh frozen & Full & 0 & 1 & 0 \\
\hline 2017 & Sohier & WES & Fresh frozen & Full & 0 & 8 & 0 \\
\hline 2018 & Høland ${ }^{a}$ & PCR and ds & Fresh frozen & 2 to 11 & 8 & 98 & 8.2 \\
\hline
\end{tabular}

References for all the articles are included in Supplementary Table S1

$P C R$ polymerase chain reaction, $d s$ direct sequencing, $b d s$ bidirectional sequencing, $C D G E$ constant denaturing gel electrophoresis, $S S C P$ singlestrand conformation polymorphism, $D G G E$ denaturing gradient gel electrophoresis, $T G G E$ temperature gradient gel electrophoresis, $W G S$ wholegenome sequencing, WES whole-exome sequencing, TS targeted sequencing

${ }^{a}$ Four publications with overlapping patient material, all patients were included in the present study (Høland, 2018)

${ }^{\mathrm{b}}$ Two publications with overlapping patient material were merged, patients that could be verified as overlapping were excluded

${ }^{c}$ Publications analyzing the tumors in patients with Li-Fraumeni syndrome, not included in calculations of mutation prevalence

${ }^{\mathrm{d}}$ Cell lines, in Nigro 1989, 88-3/14, in Legius 1994, NF88-2, NF89-11, NF90-8; in Sonobe 2000, HS-SCH-2 (established in study); in Beert, HM1-3, HM9, and HM22-23 (established in study)

${ }^{\mathrm{e}}$ Tumors from unique patients: each patient was only counted once as long as the information was available

${ }^{f}$ Only given for studies included in calculations of the overall mutation prevalence

${ }^{g}$ WGS samples were frozen, while TS samples were formalin-fixed 
carriers have a 6-13\% life time risk of developing malignant peripheral nerve sheath tumor $[2,3]$.

Malignant peripheral nerve sheath tumors typically have numerous DNA copy number aberrations including deletions of $C D K N 2 A$ and $N F 1$ and gains of EGFR, TOP2A, and BIRC5 [4-12]. Like malignant melanomas, malignant peripheral nerve sheath tumors are derived from the cells of the neural crest lineage, and both malignant peripheral nerve sheath tumors and melanomas exhibit constitutive activation of RAS signaling, mainly through $N F 1$ or $B R A F$ mutations, respectively. However, while melanomas have a high genome-wide mutation frequency [13], malignant peripheral nerve sheath tumors typically have few mutations at the nucleotide level $[14,15]$. Recurrent mutations in malignant peripheral nerve sheath tumors have only been found in a handful of genes including NF1, TP53, TERT, SUZ12, EED, and SPTBN2 [14-19].

The importance of aberrations in the tumor suppressor gene TP53 in cancer has been widely documented. In fact, the TP53 network has been suggested to be dysfunctional in most human tumors [20]. In malignant peripheral nerve sheath tumor, the first TP53 point mutation was reported in a patient with neurofibromatosis type 1 in 1989 [21]. A decade later, two independent laboratories found that concurrent knockout of one allele of each of the two genes NF1 and TP53 in mouse models resulted in the formation of tumors similar to malignant peripheral nerve sheath tumors $[22,23]$. These tumors developed when the mutated alleles were located on the same chromosome (in cis), followed by loss of the remaining copy of chromosome 17 , and resulting in loss of heterozygosity and complete loss of function of both gene products. Today, more than 30 articles on TP53 mutations in malignant peripheral nerve sheath tumors have been published (Table 1; Supplementary Table S1), still the prevalence and clinical impact of TP53 mutations in malignant peripheral nerve sheath tumor remain inconclusive due to the limited number of samples available for analyses of this rare cancer type.

In addition to aberrations targeting the TP53 gene itself, the TP53 network can be dysregulated by the changes in several other genes including amplification of $M D M 2$ or inactivating alterations of $C D K N 2 A$ [20]. MDM2 promotes tumor formation by targeting TP53 for proteasomal degradation, and $C D K N 2 A$ encodes two distinct tumor suppressor proteins $\mathrm{p} 14^{\mathrm{ARF}}$ and $\mathrm{p} 16^{\mathrm{INK} 4 \mathrm{a}}$, both acting to induce cell cycle arrest; the $\mathrm{p} 14^{\mathrm{ARF}}$ isoform interacts with and sequesters MDM2, thereby stabilizing TP53 [24].

In this study, we analyzed the genomic aberrations of TP53 and MDM2 in relation to clinical end-points in a series of more than 100 malignant peripheral nerve sheath tumors from patients with and without neurofibromatosis type 1 . For interpretation of the downstream effects of the various genomic aberrations in the TP53 network, we analyzed a TP53-mutant signature at the gene expression level.

\section{Materials and methods}

\section{Literature survey}

A search on NCBI PubMed [25] was performed to capture articles that included analyses of TP53 mutations in malignant peripheral nerve sheath tumors (Fig. 1), and a detailed overview of the identified publications, the methods used, and the specific TP53 mutations found in these studies are given in Table 1 and Supplementary Table S1.

\section{Patients and samples}

The included patients were treated between 1980 and 2010 at four European sarcoma centers: the Norwegian Radium Hospital, Oslo, Norway; Skåne University Hospital, Lund, Sweden; the University Medical Centre of Groningen, The Netherlands; and the Istituto Ortopedico Rizzoli, Bologna, Italy. The sample biobanks and research protocols were approved by the regional/local ethics committees of each participating country following informed consent from the patients. The multicenter samples and protocols used in the present study were approved by the Regional Committee for Medical and Health Research Ethics, South East Norway [2010/223/REKsør-øst].

Malignant peripheral nerve sheath tumors were collected from 50 patients with sporadic disease (median age at diagnosis 48 years) and 50 patients with neurofibromatosis type 1 (median age 29 years). None of the malignant peripheral nerve sheath tumors were radiation-induced and two were malignant triton tumors. In total, 143 tumor samples have been investigated, including 105 malignant peripheral nerve sheath tumors from 100 patients and 38 neurofibromas, of which different subsets were included in different analyses based on the available material and sample types. Blood samples were available from 18 malignant peripheral nerve sheath tumor patients. The clinical data are summarized in Table 2, while Supplementary Tables S2 and S3 provide an overview of the clinical and the molecular data for individual malignant and benign tumors, respectively.

The malignant peripheral nerve sheath tumor diagnoses were determined by sarcoma pathologists at specialized sarcoma centers in each country, and all tumors were diagnosed according to the established criteria [26]. In addition, the neighboring tissue sections of all frozen tumor samples used for DNA and RNA extraction were morphologically evaluated, and the tumor cell percentage was visually estimated to a median of $\sim 100 \%$ (interquartile range: $10 \%$ ). 


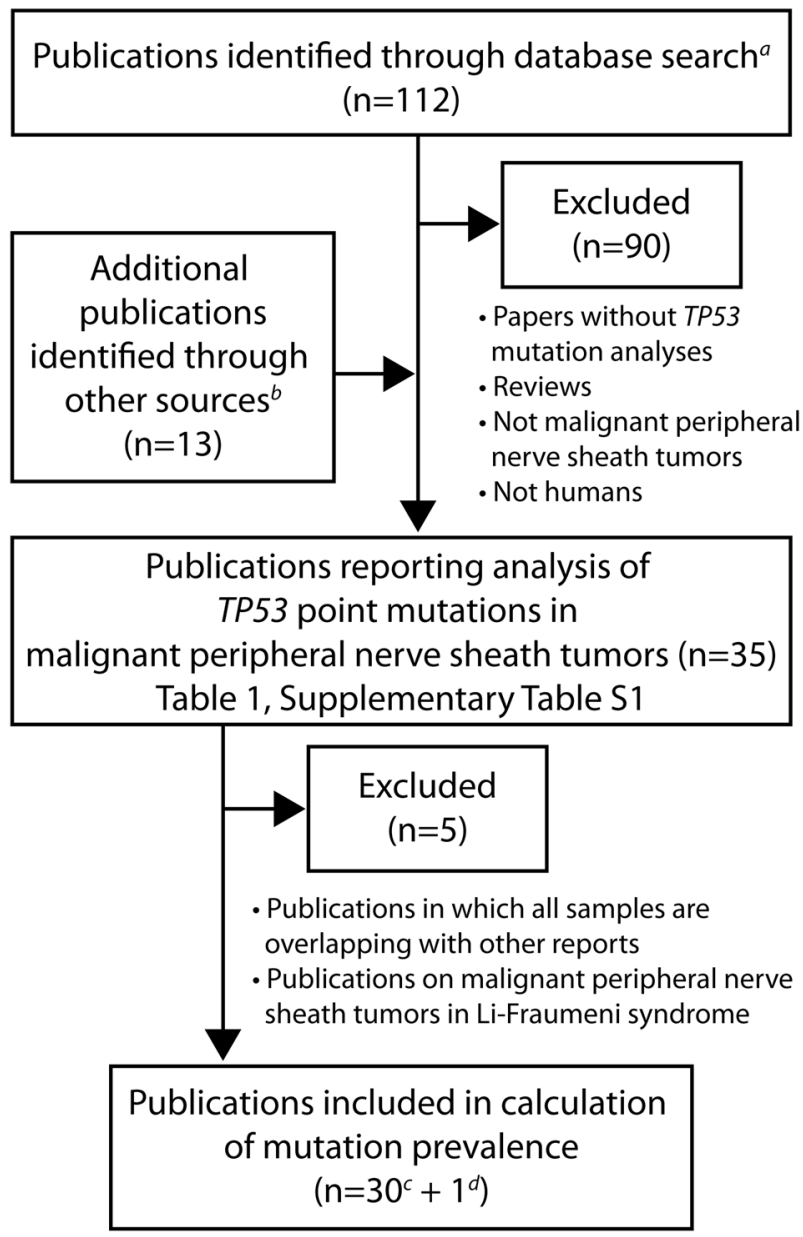

Fig. 1 Literature survey flow diagram. a Search string on NCBI PubMed: "(tp53 OR p53) AND (mpnst OR (malignant peripheral nerve sheath) OR neurofibrosarcomas OR neurofibrosarcoma OR (malignant schwannomas) OR (malignant schwannoma) OR neurosarcoma OR neurosarcomas) AND (mutation or mutations or polymorphism)". b Publications not captured by the search string. c Two of the 31 publications had overlapping tumor material and were merged into one study before calculations. d The present study

Despite their limitations in sensitivity and specificity for diagnosis of malignant peripheral nerve sheath tumor, S100 and SOX10 are markers of neural crest differentiation [27]. From the gene expression data we observed, in agreement with previous publications [27, 28], lower expression of both S100B and SOX10 in malignant peripheral nerve sheath tumors, compared to neurofibromas $\left(P=3 \times 10^{-8}\right.$ and $P=3 \times 10^{-8}$, respectively, Mann-Whitney-Wilcoxon, Supplementary Figure S5a-b), although a few malignant tumors have expression at the level of the benign tumors (Supplementary Figure S5c). The expression of S100A1 was low in both the malignant peripheral nerve sheath tumors and the benign neurofibromas (Supplementary Figure S5d), supporting no inclusion of erroneous diagnosed malignant melanomas [29].
Loss of trimethylation of lysine 27 of histone H3 (H3K27m3) has been found to discriminate malignant peripheral nerve sheath tumors from benign neurofibromas (Supplementary Figure S6a) [30, 31]. We used gene set enrichment analyses to evaluate the loss of $\mathrm{H} 2 \mathrm{~K} 27 \mathrm{~m} 3$ in our tumor material (Supplementary Table S2, S3, and S7). In agreement with previous publications [30, 31], we found that malignant tumors had significantly higher gene set scores, and therefore expectedly a higher degree of H3K27m3 loss than the benign neurofibromas ( $P=0.0009$, Mann-Whitney-Wilcoxon, Supplementary Figure S6b), further supporting the true malignant peripheral nerve sheath tumor diagnoses.

\section{DNA and RNA isolation}

Two different methods were used for DNA and RNA isolation. Either the tumor tissue was homogenized using TissueRuptor, and DNA and RNA was extracted using the Allprep DNA/RNA/miRNA Universal Kit, as recommended by the manufacturer (QIAGEN, Hilden, Germany), or the tumor tissue was manually ground in liquid nitrogen using a mortar and pestle before separate isolation of RNA and DNA. DNA was extracted using a semi-automatic phenol-chloroform extraction method, followed by ethanol precipitation in a 340A Nucleic Acid Extractor (Applied Biosystems, Foster City, CA, USA), while RNA was isolated from the tissue samples by standard Trizol methodology in $1 \mathrm{ml}$ Trizol (Invitrogen, Carlsbad, CA, USA), followed by phase separation by chloroform and precipitation using 2-propanol. The RNA pellets were dissolved in RNase free water at $55^{\circ} \mathrm{C}$. Quantity and quality measurements were carried out using UV spectrometry (NanoDrop ND-1000, Thermo Fisher Scientific, Waltham, MA, USA) and Agilent 2100 Bioanalyzer (Agilent Technologies, Santa Clara, CA, USA). All RNA samples had a RIN value above 6 (average 8.5).

\section{Mutation analyses}

The full coding sequence of TP53, exons 2-11 (393 codons, Ensembl transcript TP53-001 [ENST00000269305.4], human assembly GRCh37) and the flanking intronic regions (10 bases downstream and upstream of the exons) was analyzed for mutations by Sanger sequencing. Genomic TP53 sequences were amplified from the DNA extracts in separate single plex (exons 2-4, exons 5-6, and exons 7-9) and multiplex (exon 10 and 11) PCR reactions, giving rise to five distinct DNA fragments. HotStarTaq DNA Polymerase or QIAGEN Multiplex PCR Kit was used, as recommended by the manufacturer (Qiagen, Hilden Germany). The PCR program included 35 cycles, an annealing temperature of $60^{\circ} \mathrm{C}$, and a 45 or $90 \mathrm{~s}$ elongation step for 
Table 2 Clinical parameters for the malignant peripheral nerve sheath tumor patient cohort

\begin{tabular}{|c|c|}
\hline Clinical parameter & Patients $(n=100)$ \\
\hline \multicolumn{2}{|l|}{ Neurofibromatosis type $1^{\mathrm{a}}$} \\
\hline No & 50 \\
\hline Yes & 50 \\
\hline \multicolumn{2}{|l|}{ Sex } \\
\hline Male & 56 \\
\hline Female & 44 \\
\hline \multicolumn{2}{|l|}{ Age at diagnosis ${ }^{\mathrm{b}}$} \\
\hline Median (range) & $36(11-82)$ \\
\hline \multicolumn{2}{|l|}{ Location } \\
\hline Extremities & 70 \\
\hline Non-extremities & 29 \\
\hline Not available & 1 \\
\hline \multicolumn{2}{|l|}{ Tumor size $(\mathrm{cm})^{\mathrm{b}}$} \\
\hline Median (range) & $10(3-34)$ \\
\hline \multicolumn{2}{|l|}{ Complete remission $^{\mathrm{b}, \mathrm{c}}$} \\
\hline Yes & 65 \\
\hline No & 17 \\
\hline Not available & 18 \\
\hline \multicolumn{2}{|l|}{ Grade $^{d}$} \\
\hline Low & 7 \\
\hline High & 90 \\
\hline Not available & 3 \\
\hline \multicolumn{2}{|l|}{ Survival } \\
\hline Median survival time (months) & 42.7 \\
\hline Number of events & 60 \\
\hline Survival data available & $96^{\mathrm{f}}$ \\
\hline Lost to follow up & 4 \\
\hline \multicolumn{2}{|l|}{ Tumors analyzed } \\
\hline Primaries & 72 \\
\hline Relapses & 25 \\
\hline Metastases & 8 \\
\hline \multicolumn{2}{|c|}{ TP53 mutation or MDM2 amplification } \\
\hline None & 76 \\
\hline TP53 point mutation & 8 \\
\hline MDM2 amplification ${ }^{\mathrm{e}}$ & 5 \\
\hline Not available & 11 \\
\hline
\end{tabular}

${ }^{a}$ Two of the included tumors were triton tumors, one from a patient with neurofibromatosis type 1 and one sporadic

${ }^{b}$ Refers to the initial primary tumor, tumor size is the maximum tumor diameter

${ }^{c}$ Patients in complete remission had wide or marginal surgical margins after removal of the primary tumor and no metastasis at diagnosis, while those not in complete remission had intralesional margins and/or metastasis at diagnosis

${ }^{\mathrm{d}}$ Grade is given for the initial primary tumor, except for 3 high grade and 2 low cases, where the grade of the analyzed tumor is given (relapse or second primary)

${ }^{\mathrm{e}}$ Amplification is defined as gain of $\geq 5$ copies, relative to the median genome-wide copy number

${ }^{\mathrm{f} O n l y} 86$ patients, from whom a primary or a local relapse were analyzed, were included in the survival analyses with molecular markers, see Supplementary Table S2 for details the singleplex or multiplex PCR reaction, respectively. The PCR products were subjected to enzymatic purification using illustra ExoProStar 1-Step kit (GE Healthcare Life Sciences, Marlborough, MA, United States), and sequencing of the purified products was performed for seven distinct fragments (exons 2-3, 4, 5-6, 7, 8-9, 10, and 11) using the BigDye Terminator v3.1 Cycle Sequencing Kit (Applied Biosystems $^{\mathrm{TM}}$, ThermoFisher Scientific, Waltham, MA, USA). Details for the primers used in the initial PCR amplification and the sequencing reaction are listed in Supplementary Table S4. The resulting sequence products were further purified using the BigDye XTerminator ${ }^{\circledast}$ Purification Kit (Applied Biosystems ${ }^{\mathrm{TM}}$ ) and subjected to sequencing using the 3730 DNA Analyzer (Applied Biosystems ${ }^{\mathrm{TM}}$ ). In silico analysis of the mutations was performed manually by two investigators using the Sequencing Analysis (v5.3.1, Applied Biosystems ${ }^{\mathrm{TM}}$ ) and SeqScape (v2.5.0, Applied Biosystems ${ }^{\mathrm{TM}}$ ) softwares. All detected mutations were confirmed by sequencing of a new independent PCR product. The bases of the TP53 sequence were reported according to the sequence of the gene, hence the reverse genomic DNA strand.

\section{DNA copy number analysis}

DNA from fresh frozen tumor samples, 91 malignant peripheral nerve sheath tumors and 28 neurofibromas, and 18 blood samples were individually, processed, and hybridized on the Genome-Wide Human SNP Array 6.0 from Affymetrix (Thermo Fisher Scientific), as described in the Affymetrix Cytogenetics Copy Number Assay User Guide (P/N 702607 Rev. 2). Raw probe intensity data from the scanned images of the arrays were stored in cell intensity files by the Affymetrix Gene Chip Command Console software (version 1.0), and quality control of the individual cell intensity files was performed using the Affymetrix Genotyping Console software (version 4.1.4.840). All samples had a cell intensity data quality above the recommended threshold (Contrast QC $>0.4$ ) for individual samples, and $>1.7$ for the dataset (Affymetrix white paper; Quality Control Assessment in Genotyping Console, September 30, 2008, Revision 1), and a Median Absolute Pairwise difference below 0.4 (Affymetrix white paper; Median of the Absolute Values of all Pairwise Differences and Quality, Control on the Affymetrix, Genome-Wide Human SNP Array 6.0, February 11, 2008, Revision 1).

For copy number analysis, the cell intensity files were preprocessed according to the PennCNV protocol [32] adapted for Affymetrix genotyping arrays [33], as previously described [34]. HapMap samples previously analyzed on the SNP Array $6.0(n=270$ individuals from four populations) [35] were used as reference for quantile normalization and calculation of $\log \mathrm{R}$ ratio and $\mathrm{B}$ allele frequency. 
Allele-specific copy number analysis was performed on the $\log \mathrm{R}$ ratio and $\mathrm{B}$ allele frequency values using the ASCAT algorithm (ASCAT version 2.3, penalty parameter 50) [36]. Discrete copy number states were decided relative to the median genome-wide copy number in each tumor sample. All genomic positions refer to genome version GRCh37 (Hg19).

For genomic identification of significant targets in cancer (GISTIC analysis) [37, 38], the $\log R$ ratio values were subjected to winsorization and single-sample segmentation by the PCF algorithm implemented in the Bioconductor package copynumber (version 1.12.0; penalty parameter gamma $=100$; minimum number of probes per segment, $k_{\min }=5$ ) [39]. Version 2.0.22 of GISTIC was used, and copy number estimates $>0.1$ were called as copy number gain, while estimates $<-0.1$ were called as loss; the broad length cut-off was set to 0.7 (-brlen 0.7), normal arbitrated peel-off was performed (-armpeel 0 ), and we calculated the significance of deletions at a gene level (-genegistic 1), otherwise default settings. The reference genome file hg19. mat and the copy number variation file CNV.hg19. bypos.111213.txt were used.

\section{Gene expression analysis}

Total RNA (100 ng) from 63 malignant peripheral nerve sheath tumors and 15 neurofibromas was used as input for cDNA synthesis, followed by amplification and DNA sense strand labeling, according to the GeneChip Whole Transcript (WT) PLUS Reagent Kit Manual (Affymetrix). Each sample was hybridized to Affymetrix GeneChip Human Transcriptome 2.0 arrays for $16 \mathrm{~h}$ and washed, stained, and scanned, as recommended. For each sample, a cell intensity file was generated by the Affymetrix GeneChip Command Console software (version 4.0). The cell intensity files intensities were adjusted by guanine cytosine count correction and signal space transformation before being further processed through background correction, quantile normalization, and summarization at the gene level by robust multiarray average using the Affymetrix Expression Console Software (v1.4.1.46, HTA2_0.r3 library files), giving data on $\log 2$ scale. All the samples passed the recommended quality control measures and no outlier samples were identified. The hta-2_0na36.hg19_transcript.csv file from Affymetrix was used for transcript cluster annotation. The dataset was filtered to include only protein-coding transcripts, and entries from the NCBI Reference Sequence Database (RefSeq) were prioritized in order to retain one transcript cluster per gene. Gene expression data on 18,618 unique genes was used as input for single-sample gene set expression enrichment analysis using the Bioconductor package GSVA (version 1.22.4, default settings) [40].

\section{Statistical analysis}

Five-year disease-specific survival was analyzed using the software package IBM SPSS version 21.0 (IBM Corporation, Armonk, NY, U.S.A.). The Kaplan-Meier method and the Cox proportional hazards model with Wald test were used to provide univariable and multivariable hazard ratios and $95 \%$ confidence intervals. In multivariable analyses, all variables were included in the proportional hazards model. Only patients with a primary malignant peripheral nerve sheath tumor or a local relapse were included in the survival analyses (Table 3; Supplementary Table S2). Five-year disease-specific survival was counted from the time of diagnosis of the primary malignant peripheral nerve sheath tumor and considering death from disease as an event. All survival analyses were also performed separately for the primary and the relapsed malignant peripheral nerve sheath tumors, and the same associations as for the primaries and relapses combined were found (data not shown).

Fisher's exact test was used to compare the frequency distributions between the groups, Mann-Whitney-Wilcoxon exact test was used to compare the differences in the distribution between the two groups of continuous variables, and Spearman's test were used to analyze the correlation for continuous and ordinal variables. All tests were two-sided, and $P$-values lower than 0.05 are reported as significant.

\section{Results}

\section{Literature survey indicates a moderate mutation prevalence of TP53 in malignant peripheral nerve sheath tumor}

Across the 31 published papers found to report TP53 mutations in malignant peripheral nerve sheath tumor (including the present study and excluding two studies on patients with the Li-Fraumeni syndrome), the TP53 mutation prevalence was $16.9 \%$ (78/462 tumors from unique patients, Table 1; Supplementary Table S1). By excluding the samples reported to be formalin-fixed and paraffin-embedded, the mutation prevalence dropped to $13.8 \%$ (43/311 tumors), compared to $23.2 \%$ (35/151 tumors) for formalin-fixed samples alone.

Totally, 85 TP53 mutations were identified in malignant peripheral nerve sheath tumors in the present study and the reviewed literature, including double mutations in three tumors and four mutations found in patients with the $\mathrm{Li}$ Fraumeni syndrome. These represent 73 unique mutations in 62 different codons of exons 4 to 9 or in intronic sequences (Fig. 2a-c). The mutation types and distribution resemble those reported for other tumor types (Supplementary Figure S1, data on somatic mutations from the IARC TP53 Database) [41], and the most recurrent 
mutation in malignant peripheral nerve sheath tumors ( $p$. $\mathrm{R} 175 \mathrm{H}$, identified in four tumors) is also one of the most recurrent mutations in cancer in general. However, the percentage of TP53 splice site mutations in malignant peripheral nerve sheath tumors was higher than in cancer in general, $8.2 \%$ versus $2.3 \%$, respectively.

\section{Only the mutated allele of TP53 remains}

Among our own 98 malignant peripheral nerve sheath tumor patients, we identified nine TP53 point mutations in eight tumors from eight different patients $(8.2 \%)$, while no TP53 mutations were identified in 35 benign tumors (Supplementary Table S2 and S3). Only two of the identified mutations have been reported in malignant peripheral nerve sheath tumors previously (p.S241F and p.R273H, Supplementary Table S1), although all have been reported in other cancers (TP53 database [41]). The mutation types included one nonsense, one splice site, and seven missense mutations (Fig. 2a-c). The nonsense mutation has been reported by IARC to affect tetramerization of the TP53 protein, while the splice site mutation is found at a consensus acceptor splice site and should affect mRNA processing. All the missense mutations have been reported to be deleterious to protein function, except for the p.V203L mutation [41]. However, this p.V203L mutation was found as a double mutant along with the deleterious p.V197E mutation in one primary malignant peripheral nerve sheath tumor. The Sanger sequencing chromatograms (Supplementary Figure S2) indicated that all mutations, except p.V203L, were hemi or homozygous, with little or nothing of the wild-type allele present.

Allele-specific copy number data confirmed that all TP53-mutated samples were homo or hemizygous for all single-nucleotide polymorphism probes in a region surrounding the TP53 locus. This supports the notion that loss of heterozygosity, either by loss or copy number neutral loss of heterozygosity, has resulted in complete loss of function of the TP53 gene in all mutated tumors.

\section{Polymorphisms in TP53}

The common non-synonymous single-nucleotide polymorphism in codon 72 of exon 4 in TP53 (c.215G > C, p.

Table 3 Survival analysis for patients with malignant peripheral nerve sheath tumor

\begin{tabular}{|c|c|c|c|c|c|c|c|c|}
\hline \multirow[t]{2}{*}{ Clinical parameter } & \multirow[t]{2}{*}{ Categories } & \multicolumn{4}{|c|}{ Univariable } & \multicolumn{3}{|c|}{ Multivariable } \\
\hline & & $N$ & $\begin{array}{l}\% \text { survival }^{\mathrm{c}} \\
\text { (standard } \\
\text { error) }\end{array}$ & $P$-value ${ }^{\mathrm{d}}$ & $\begin{array}{l}\text { Hazard ratio }(95 \% \\
\text { confidence } \\
\text { interval })\end{array}$ & $N$ & $P$-value ${ }^{\mathrm{d}}$ & $\begin{array}{l}\text { Hazard ratio }(95 \% \\
\text { confidence } \\
\text { interval })\end{array}$ \\
\hline \multirow[t]{2}{*}{ Neurofibromatosis type 1} & No & 44 & $55.1(7.7)$ & Ref & & 37 & Ref & \\
\hline & Yes & 42 & $35.6(7.7)$ & 0.06 & $1.79(0.99-3.23)$ & 33 & 0.78 & $0.90(0.41-1.96)$ \\
\hline \multirow[t]{2}{*}{ Sex } & Male & 49 & $48.4(7.2)$ & Ref & & 37 & Ref & \\
\hline & Female & 37 & $41.8(8.2)$ & 0.41 & $1.28(0.71-2.30)$ & 33 & 0.97 & $1.02(0.47-2.21)$ \\
\hline Age at diagnosis ${ }^{\mathrm{a}, \mathrm{b}}$ & & 86 & 0.99 & $\begin{array}{l}1.00 \\
(0.98-1.02)\end{array}$ & 70 & 0.15 & $\begin{array}{l}0.98 \\
(0.96-1.01)\end{array}$ & \\
\hline \multirow[t]{2}{*}{ Location } & Extremities & 63 & $53.4(6.5)$ & Ref & & 54 & Ref & \\
\hline & $\begin{array}{l}\text { Non- } \\
\text { extremities }\end{array}$ & 23 & $26.6(9.6)$ & 0.02 & $2.05(1.11-3.78)$ & 16 & 0.34 & $1.51(0.65-3.54)$ \\
\hline Tumor size $\mathrm{a}^{\mathrm{a}, \mathrm{b}}$ & & 83 & & 0.01 & $1.06(1.01-1.11)$ & 70 & 0.03 & $1.06(1.01-1.12)$ \\
\hline \multirow[t]{2}{*}{ Complete remission ${ }^{\mathrm{a}, \mathrm{e}}$} & Yes & 61 & $57.4(6.5)$ & Ref & & 56 & Ref & \\
\hline & No & 14 & $7.9(7.5)$ & $4.1 \times 10^{-5}$ & $4.51(2.29-9.25)$ & 14 & 0.002 & $3.71(1.63-8.46)$ \\
\hline \multirow{2}{*}{$\begin{array}{l}\text { TP53 mutation or MDM } 2 \\
\text { amplification }\end{array}$} & No & 68 & $54.3(6.2)$ & Ref & & 60 & Ref & \\
\hline & Yes & 13 & $0(58)^{\mathrm{f}}$ & $3.0 \times 10^{-4}$ & $3.52(1.78-6.98)$ & 10 & 0.01 & $3.39(1.38-8.33)$ \\
\hline
\end{tabular}

Values written in bold phase indicate significant findings

${ }^{\text {a }}$ Refers to the initial primary tumor, tumor size is the maximum tumor diameter $(\mathrm{cm})$

${ }^{\mathrm{b}}$ Continuous variable

${ }^{\mathrm{c}}$ Cumulative Proportion Surviving

${ }^{\mathrm{d}} P$-values from Wald test, Cox regression for 5 years disease-specific survival

${ }^{\text {e}}$ Patients in complete remission had wide or marginal surgical margins after the removal of the primary tumor and no metastasis at diagnosis, while those not in complete remission had intralesional margins and/or metastasis at diagnosis

${ }_{\mathrm{f}}^{\mathrm{f}}$ or groups with no survivors, the value in parentheses indicates months of the last disease-specific death 


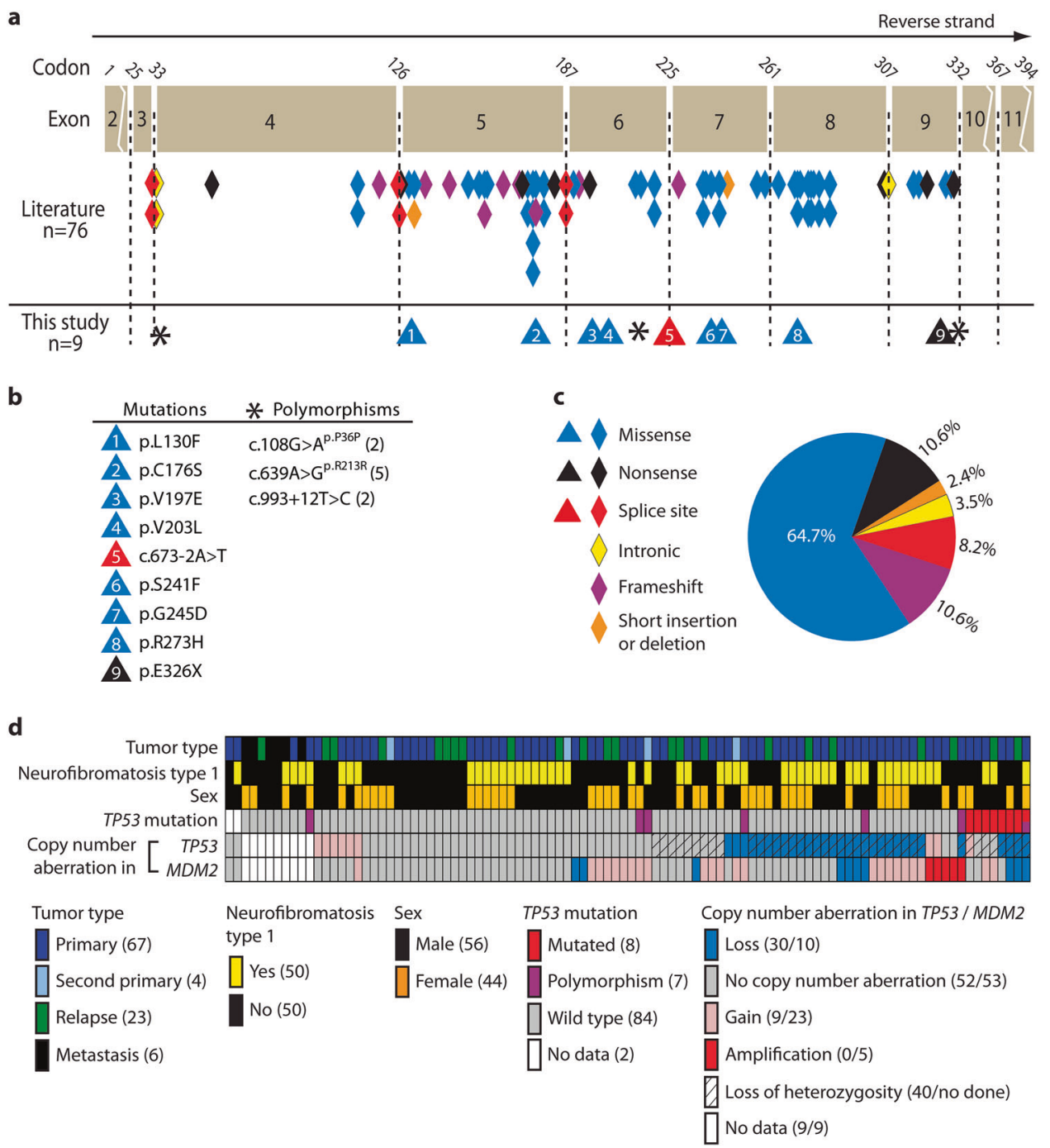

Fig. 2 Genetic aberrations in TP53 and MDM2 identified in malignant peripheral nerve sheath tumors. a Exon/intron distribution of mutations. Diamond shaped ( $\$$ ): 76 TP53 mutations identified in malignant peripheral nerve sheath tumors from 74 patients from the literature (4 patients with Li-Fraumeni syndrome). Triangle shaped ( $\mathbf{\Delta})$ : Nine mutations from eight malignant peripheral nerve sheath tumors identified in this study. Star shaped (*): identified polymorphisms. b Mutations and polymorphisms identified in the present study. Polymorphisms were identified in malignant peripheral nerve sheath tumors from more than one patient, numbers are given in parentheses, c. $108 \mathrm{G}>\mathrm{A}$ and c. $993+12 \mathrm{~T}>\mathrm{C}$ were found in overlapping samples. $\mathrm{c}$ Color coding for different mutation types and pie chart showing the percentage of mutation types identified from the literature and the

R72P, rs1042522) was also examined in the Sanger sequencing data, and 15 malignant peripheral nerve sheath tumors were regarded as homozygous $\mathrm{C} / \mathrm{C}$ allele carriers, 24 as heterozygous, and 58 as homozygous $\mathrm{G} / \mathrm{G}$ carriers (Supplementary Table S2). The calculated G allele frequency across malignant peripheral nerve sheath tumors from 97 patients was $72 \%$ (considering heterozygous samples as $50 \% \mathrm{C}$ and $\left.\mathrm{G}\left(n_{(\mathrm{C} / \mathrm{G})}+2 n_{(\mathrm{G} / \mathrm{G})}\right) / 2 n_{(\text {Total })}\right)$, in good present study. d Overview of the patients (numbers given in parentheses), clinical data, and genetic aberrations found in TP53 and MDM2. One malignant peripheral nerve sheath tumor had both, a mutation and a polymorphism. For patients where more than one malignant tumor was analyzed, only the primary or second primary tumor is given. Copy number is relative to the genome-wide median copy number state (nAB $\div$ median copy number). Gain of $\geq 5$ is defined as amplification (range: 5-23). Minimal region of overlap with copy number aberration: $61.3 \mathrm{kB}$ for TP53 (chr17: 7,542,659-7,603,944); $396 \mathrm{kB}$ for MDM2 (chr12: 69,127,774-69,523,923). Minimal region of loss of heterozygosity covering the TP53 locus: $11.4 \mathrm{MB}$, 4170 single nucleotide polymorphism probes

concordance with the European (non-Finnish) samples included in the Exome Aggregation Consortium database [42].

We also identified the less common intragenic single nucleotide polymorphism variants (Fig. 2; Supplementary Tables S2 and S3) including c.639A $>G^{\text {p.R213R }}($ rs 1800372) in five malignant peripheral nerve sheath tumors and one neurofibroma, as well as c.108G $>\mathrm{A}^{\mathrm{p} . \mathrm{P} 36 \mathrm{P}}(\mathrm{rs} 1800370)$ and 
c. $993+12 \mathrm{~T}>\mathrm{C}$ (rs1800899) found in overlapping samples including three malignant tumors from two patients and two neurofibromas.

\section{Increased mutation frequency by integration of copy number aberrations and LOH at the TP53 locus}

Copy number aberration and zygosity at the TP53 locus were analyzed in malignant peripheral nerve sheath tumors from 91 patients (Fig. 2d; Supplementary Table S2), and the loss of one or two copies of the gene was found in 30 malignant tumors $(33 \%)$, however, because almost half of the tumors had a median genome-wide copy number of three or higher, none of these tumors had complete loss of the TP53 gene. Point mutations co-occurred with copy number loss in four patients. Of the 30 malignant peripheral nerve sheath tumors with loss, 20 were neurofibromatosis type 1-associated and 10 where sporadic cases $(P=0.044$, Fisher's exact test, $n=91)$. For tumors with copy number gain $(n=9,10 \%)$, the affected region was not limited to the TP53 gene sequence, but included all or most of chromosome 17, suggesting that TP53 was not a specific target gene for these aberrations. Loss of heterozygosity at the TP53 locus was found in $40(44 \%)$ malignant peripheral nerve sheath tumors, of these, 12 cases (13\%) were copy number neutral, which co-occurred with point mutations in three cases. Altogether, the malignant peripheral nerve sheath tumors from 51 patients $(57 \%)$ had genetic aberrations in TP53 (point mutation and/or copy number aberration), these TP53 copy number changes were found to be tumor-specific, as no aberrations were found in 18 blood samples from malignant peripheral nerve sheath tumor patients, nor in 28 neurofibromas.

\section{Amplification of the MDM2 locus affects malignant peripheral nerve sheath tumors with non-aberrant TP53}

Five of the 91 malignant peripheral nerve sheath tumors had amplification of the MDM2 gene (defined as gain of five or more copies) (Fig. 2d). MDM2 amplification and TP53 point mutations were mutually exclusive, while $M D M 2$ amplification and loss of the TP53 locus were nearly mutually exclusive (co-occurrence in one tumor). GISTIC analysis [38] showed that MDM2 $(\operatorname{chr} 12: 69127775-69649624 ; q$-value $=$ $\left.1.32 \times 10^{-5}\right)$ and TP53 (chr17:7,479,693-7,613,707; $q$-value $=0.075)$ were contained within the focal regions with aberrant and frequent gains and losses in malignant peripheral nerve sheath tumors, respectively, suggesting that $M D M 2$ and TP53 were the possible target genes for the copy number aberrations. No malignant peripheral nerve sheath tumors had amplification of the MDM4 gene, a homolog of MDM2.

\section{Poor prognostic associations of genetic aberrations in TP53 and MDM2}

For patients with sporadic malignant peripheral nerve sheath tumor, the point mutation in TP53 was associated with a higher age at diagnosis ( $P=0.01$, Mann-Whitney-Wilcoxon test), with a median age of 71 years for patients with mutation $(\mathrm{n}=5)$ versus 43 years for patients without mutation $(n=$ $44)$, this association was not found for the neurofibromatosis type 1 patients.

TP53 mutation was associated with a poor survival for the complete patient group, and all patients with the mutation died of the disease within 5 years (median time to death 19 months), compared to $49 \%$ (median time to death 42 months) of patients without the mutation (hazard ratio $=$ 3.2, confidence interval: 1.44-6.88, $P=0.04, \mathrm{n}=84$, Supplementary Figure S3a). The combined analysis of TP53 mutation and MDM2 amplification data revealed an even stronger prognostic impact, independent of the clinical parameters in multivariable analysis (Table 3). Moreover, a total of $60 \%$ of the patients (53/89, Fig. 2d) had a genetic aberration in either TP53 (point mutations, copy number aberration, and/or loss of heterozygosity) or MDM2 (amplification), and this was significantly associated with an inferior 5-year disease-specific survival rate (Fig. 3a; Supplementary Figure S3a-e). The same association was observed for patients believed to be in complete remission after surgery $(n=57)$ (Fig. 3b), and in separate analyses of neurofibromatosis type 1 -associated and sporadic cases (Supplementary Figure S3g-h).

There was also an association between the singlenucleotide polymorphism at codon 72 of TP53 (c.215G > C, p.R72P) and patient survival (Supplementary Figure S3f), and the single nucleotide polymorphism was an independent prognostic marker in multivariable analysis including clinical parameters (neurofibromatosis type 1 association, sex, age at diagnosis, primary tumor size and location, and complete remission status). Compared to the homozygous $\mathrm{G} / \mathrm{G}$ allele carriers $(n=43)$, the heterozygous $(\mathrm{G} / \mathrm{C})$ allele carriers $(n=20)$ had a reduced risk of dying of the disease (multivariable hazard ratio $=0.49$, confidence interval: $0.19-1.26, P=0.14$ ), while the homozygous $\mathrm{C} / \mathrm{C}$ allele carriers $(n=8)$ had a significantly higher risk (multivariable hazard ratio $=2.7$, confidence interval: 1.02-7.128, $P=0.046$ ). We found an association between the codon 72 polymorphism and the genetic aberrations found in TP53 $(P=0.003$, Fisher's exact test, $n=88)$, where $93 \%(13 / 14)$ of tumors homozygous for $\mathrm{C}$ were affected by aberration in TP53 (point mutations, copy number aberration and/or loss of heterozygosity), compared to $55 \%(29 / 53)$ of the tumors homozygous for $\mathrm{G}$ and $38 \%$ $(8 / 21)$ of the heterozygous tumors. 
For the less common single nucleotide polymorphism variants, c.639A $>\mathrm{G}^{\text {p.R213R }}$, c. $108 \mathrm{G}>\mathrm{A}^{\text {p.P36P }}$, and c. $993+$ $12 \mathrm{~T}>\mathrm{C}$, there were no association with prognosis.

\section{Genetic aberrations define a common, poor prognostic "TP53-mutated phenotype" at the transcriptome level}

The genomic aberrations of TP53 and MDM2 were analyzed in relation to gene expression data in 63 malignant peripheral nerve sheath tumors and 15 neurofibromas to reveal the transcriptional consequences on the TP53 network. As anticipated, the TP53 expression level was lower in tumors with the copy number loss of the TP53 gene ( $n=14)$, compared to tumors without the loss $(n=42)$ (difference in median on $\log 2$-scale $=0.8, P=0.01$, Mann-Whitney-Wilcoxon test), and tumors with TP53 mutation $(n=4)$ did not have a significantly different TP53 expression level than tumors without the mutation ( $n=56$, Supplementary Figure S4a). MDM2 gene expression was positively correlated with the discrete copy number of the MDM2 gene (Spearman's prho $=0.51, P=3 \times 10^{-5}, \mathrm{n}=60$, Supplementary Figure S4b), and a particularly high expression was found in tumors with high-level amplification. Furthermore, TP53 and MDM2 gene expression were positively correlated (Spearman's $\rho=0.30, P$ $=0.007, n=63$, Supplementary Figure S4c), however, in contrast to $T P 53, M D M 2$ gene expression was significantly lower in TP53-mutated $(n=4)$, compared to wild-type tumors $(n=58)$ (difference in median on $\log 2$-scale $=1.71, \quad P=0.002$, Mann-Whitney-Wilcoxon test, Supplementary Figure S4d).

We performed single-sample gene set expression enrichment analysis for a set of 15 genes previously found to be upregulated in breast tumors with point mutations in the TP53 gene (Supplementary Table S5) [43]. Significant associations were found between the gene set scores and genetic aberrations, with highest scores in samples with TP53 point mutation or MDM2 amplification, followed by the copy number aberration and/or loss of heterozygosity at the TP53 locus, thus indicating a common "TP53-mutated phenotype" resulting from either type of genomic aberration (Fig. 3c-e). Moreover, neurofibromas $(n=15)$, none of which had genetic aberrations in these two genes, had significantly lower gene set scores than the malignant tumors $(n=63) \quad\left(P=3 \times 10^{-10}\right.$, Mann-Whitney-Wilcoxon test $)$. For malignant peripheral nerve sheath tumors without genetic aberrations in TP53 or MDM2, there was a trend that those with the loss of the $C D K N 2 A$ gene $(n=15)$ had a higher gene set score than those without the loss $(n=13)$ $(P=0.11$, Mann-Whitney-Wilcoxon test $)$.

Gene expression of TP53 or MDM2 alone was not associated with patient survival, however, the gene set analysis confirmed the prognostic relevance of the TP53 network, with increasing risk according to a continuously increasing TP53 gene set score (hazard ratio $=4.1$, confidence interval: $1.73-9.79, P=0.001, n=60)$. There was a bimodal distribution of the gene set score across the samples, and stratification of the patients (Fig. 3e,f) revealed a 5-year disease-specific survival rate of $39 \%$ for the highscore group, compared to $75 \%$ for the low-score group ( $P$ $=0.01)$. This prognostic relevance was retained among the subset of patients who were in complete remission (hazard ratio $=4.4$, confidence interval: 1.57-12.1, $P=0.005$, $n=48)$.

By estimating the PRC2 loss using the gene set enrichment analyses, we confirm that loss of $\mathrm{H} 2 \mathrm{~K} 27 \mathrm{~m} 3$ is associated with inferior survival for malignant peripheral nerve sheath tumor patients (Supplementary Figure S6c). However, in multivariable analyses, the TP53 gene set signature had the strongest prognostic impact (Supplementary Figure S6e), and dysregulation of both biological processes identified a patient subgroup $(n=25,42 \%)$ with particularly poor survival (hazard ratio $=9.1$, confidence interval:2.07-39.9, $P=0.003$, Supplementary Figure S6d).

\section{Discussion}

TP53 is one of few recurrently mutated genes in malignant peripheral nerve sheath tumor. Here, we report the largest study on genomic aberrations in TP53 and its negative regulator $M D M 2$ published to date for this cancer type. We confirm that point mutations in TP53 and amplification of MDM2 are mutually exclusive; but while each of these aberrations have moderate frequency alone, integrated gene expression analyses reveal a common and poor prognostic "TP53 mutant phenotype" in $60 \%$ of the patients.

The survey of the literature on TP53 mutations in malignant peripheral nerve sheath tumors revealed a mutation prevalence of $16.9 \%$ across 462 tumors (Table 1), clearly establishing that TP53-mutated tumors constitute a significant subgroup of malignant peripheral nerve sheath tumors. In our own patient cohort, we found mutations in $8.2 \%$. The higher prevalence found in the literature may in part be explained by the inclusion of mutation data from formalin fixed and paraffin embedded samples, which are known to give rise to higher numbers of false positive mutation calls. Furthermore, the literature overview contains several single case reports with TP53-mutated tumors, suggesting that case reports with wild-type samples are underrepresented. However, excluding studies with a small sample size did not have a profound effect on the overall mutation prevalence.

All mutated tumors had loss of heterozygosity at the TP53 locus, indicating that biallelic inactivation of TP53 is an important event in a subgroup of malignant peripheral nerve sheath tumors. However, consistent with our previous results [44], such events are rare. The dominant negative 


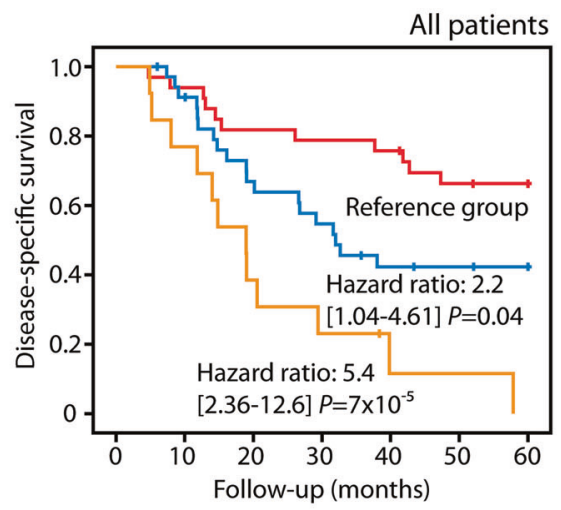

Number at risk in each subgroup

$\begin{array}{rcccccc}33 & 31 & 27 & 26 & 25 & 21 & 20 \\ -35 & 31 & 22 & 18 & 13 & 12 & 11 \\ 13 & 10 & 5 & 3 & 1 & 1 & 0\end{array}$

C

Subgroups

(numbers included in the gene expression analysis)

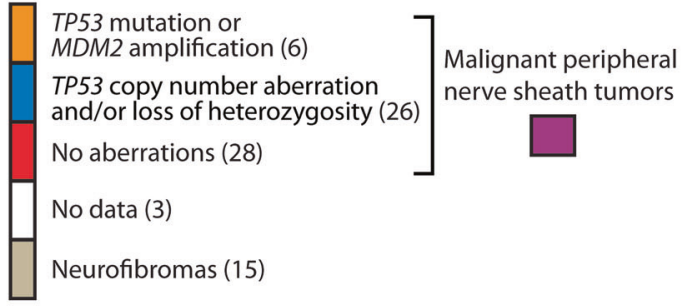

e

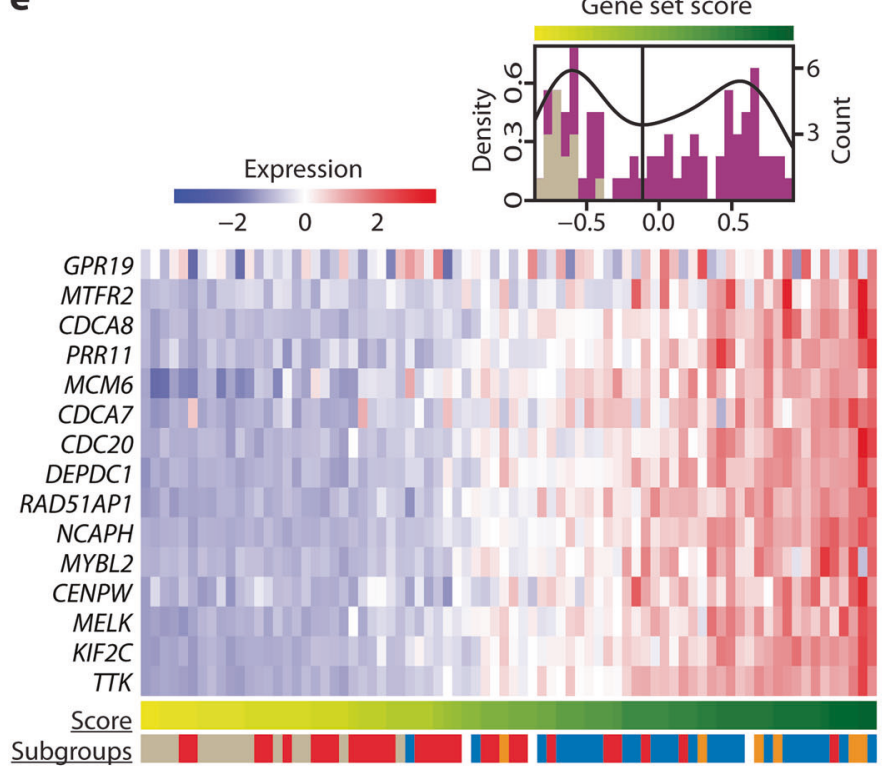

b

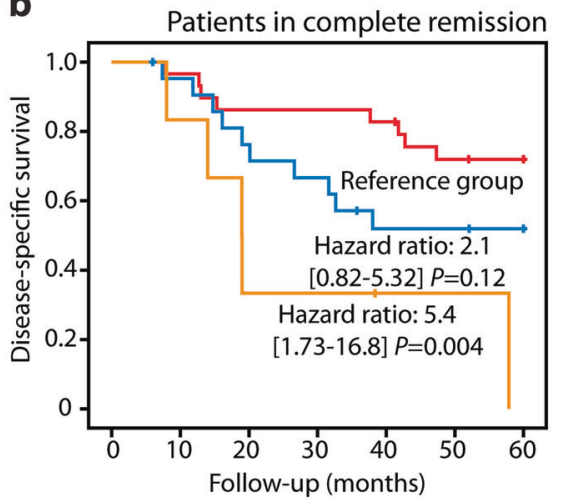

Number at risk in each subgroup

$\begin{array}{ccccccc}29 & 28 & 25 & 25 & 24 & 20 & 19 \\ 22 & 20 & 16 & 14 & 10 & 10 & 9 \\ 6 & 5 & 2 & 2 & 1 & 1 & 0\end{array}$

d

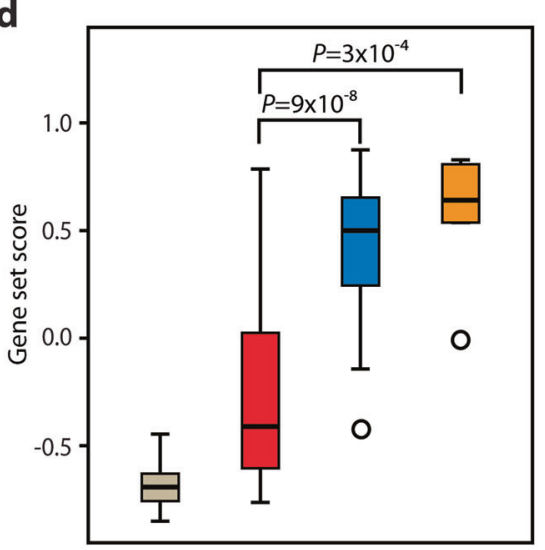

Subgroup

f
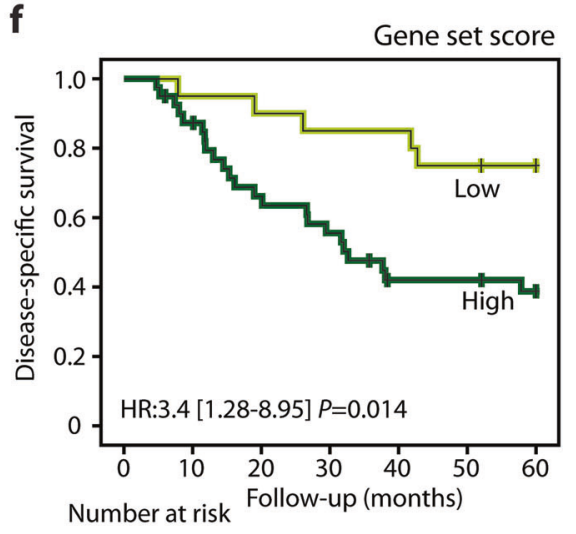

$\begin{array}{lllllll}20 & 19 & 18 & 17 & 17 & 15 & 14\end{array}$
Fig. 3 Dysregulation of the TP53 network in malignant peripheral nerve sheath tumors and association to patient survival. a Kaplan-Meier curves for patients in different molecular subgroups for all patients $(n=81)$ and $\mathbf{b}$ patients in complete remission $(n=57)$. Hazard ratios, $95 \%$ confidence intervals (in brackets), and $P$-values are from Wald test. c Color codes for the different subgroups. d Box plot illustrating the difference in gene set score for each molecular subgroup, $P$-values from Mann-Whitney-Wilcoxon exact test. e Heatmap shows the expression of the 15 genes in the gene set for each individual sample $(n=63, \log 2$ expression values for each gene are scaled to mean 0 and standard deviation 1 , samples are sorted according to the gene set score). The graph above the heatmap shows the Kernel density estimation curve and histogram for the amounts of samples with the given gene set score, a vertical line is drawn at the minimum point of the density curve $(-0.12)$. f Kaplan-Meier curves for patients with a high $(\geq-0.12)$ and low $(<-0.12)$ gene set score 
effect of TP53 mutation may indicate that biallelic inactivation is redundant to the malignant cells. A high percentage of TP53 mutations in malignant peripheral nerve sheath tumors, and in cancer in general, are missense mutations in exons 4 to 9 that affect the DNA binding domain and create full-length protein subunits that can form non-functional wild-type/mutant heterotetramers. However, as stated by Rivlin et al. [45], the heterozygous state is often transient, and TP53 mutations are frequently followed by loss of heterozygosity during cancer progression, implying that the dominant negative effect is not sufficient to inactivate the wild-type protein.

We found that one third of malignant peripheral nerve sheath tumors had loss of a chromosomal region containing TP53 as a possible gene target, as determined by GISTIC analysis. However, no tumors had complete deletion of the TP53 locus, in good concordance with the study by Lee et al. [14]. As others have also suggested previously [46, 47], our data imply that haploinsufficiency of TP53 also contributes to malignant peripheral nerve sheath tumor formation. Studies in other cancer types have shown TP53 methylation as a mechanism for allele inactivation [48-51], however, methylation of TP53 in malignant peripheral nerve sheath tumor has so far not been identified [46, 52]. Furthermore, in mouse models one mutated Tp53 allele, in combination with mutation in the $N f 1$ gene, trigger malignant peripheral nerve sheath tumor development [22, 23, 47]. Only $20 \%$ of malignant peripheral nerve sheath tumors with loss of heterozygosity at the TP53 locus were shown to have a mutation in the remaining allele. In most cases, the loss of heterozygosity region included the whole chromosome arm, and therefore, TP53 cannot be singled out as the main target for these changes. Nevertheless, we observed a poorer prognosis for patients with loss of heterozygosity of the TP53 locus in their tumors.

As shown in the current and previous studies, a subgroup of malignant peripheral nerve sheath tumors without TP53 mutation had amplification of the MDM2 gene [53, 54]. Tumors with mutation in TP53 also had lower expression of $M D M 2$, which underlines the mutually exclusive effect of these genetic events. In total, $60 \%$ of the patients had aberrations in either TP53 or MDM2, showing that the TP53 network is targeted in a much higher fraction of malignant peripheral nerve sheath tumors than can be explained by point mutations in TP53 alone. Further highlighting the importance of the TP53 network, expression analysis of a gene set previously found to be associated with TP53 point mutation in breast tumors [43], revealed a significant association with TP53/MDM2 genetic aberrations also in malignant peripheral nerve sheath tumors. This suggests similar consequences of the different types of mutations at the transcriptome level and therefore a common "TP53 mutated phenotype". The majority of the genes in this expression signature have been shown to have roles in cellular processes relevant for tumor development (cell cycle progression, apoptosis, DNA damage response), and the poor patient survival associated both with TP53/MDM2 genetic aberrations and the gene expression signature reinforces a clinical importance of the "TP53 mutated phenotype" in malignant peripheral nerve sheath tumor.

Loss of $\mathrm{H} 3 \mathrm{~K} 27 \mathrm{~m} 3$ has been found to differentiate malignant peripheral nerve sheath tumor from benign neurofibromas [30, 31] and to identify a patient subgroup with poor survival [31]. The Polycomb repressive complex 2 (PRC2) establishes and maintains the di- and trimethylation of $\mathrm{H} 3 \mathrm{~K} 27$, and it has been shown that malignant peripheral nerve sheath tumors with homozygous loss of PRC2 components have complete loss of H3K27me3 [14, 15]. Using gene set enrichment analyses, we found an inferior survival among malignant peripheral nerve sheath tumor patients with dysregulated PRC2 activity; nevertheless, the TP53 gene set still added prognostic information and this signature was an even stronger predictor of survival than the PRC2 gene set.

Somatic or germline single nucleotide polymorphisms may affect the patients' risk for developing a tumor or progression of the disease. Indeed, we and others have found that patients with a proline residue at codon 72 of TP53 had worse outcome than patients with an arginine residue, or a heterozygous genotype [55]. The difference could be explained by the higher frequency of genetic aberrations in TP53 (point mutations, copy number aberration and/or loss of heterozygosity) among the tumors with a codon 72 proline.

In malignant peripheral nerve sheath tumor cell lines we have observed [56], as anticipated, that drugs targeting MDM2 induced proteasomal degradation of TP53, e.g., serdemetan and Nutlin-3, have little effect in TP53 mutant cell lines, as compared to TP53 wild-type cell lines and normal controls. However, we found that AZ3146, a drug against the protein kinase TTK, also known as MPS1 (Monopolar spindle 1), one of the genes in the 15-gene TP53 signature set, gave a moderate, but consistently higher effect in seven malignant peripheral nerve sheath tumor cell lines compared to bone marrow and normal Schwann cells. This suggests that TTK could be a target for treatment of the TP53 inactivated tumors, which also have a high expression of TTK (Fig. 3e). TTK has been identified as a potential diagnostic, as well as prognostic, marker and as a target for therapy in several malignancies, including melanoma and glioblastoma [57]. A handful of clinical trials involving TTK inhibition have been conducted. Interestingly, one of these trials (ClinicalTrials.gov identifier NCT00676949) used peptide vaccines against TTK in combination with four other epitopes highly expressed in solid tumors, of which two were also related to the TP53 signature gene set, 
DEPDC1 and KIF20B (as KIF2C a member of the kinesin superfamily), and this clinical trial showed a significant association between the treatment and longer survival [58].

In conclusion, the survey of the literature and the findings from our own patient cohort confirm the importance of TP53 point mutations in a subpopulation of malignant peripheral nerve sheath tumors. Integrated analysis with copy number aberrations and gene expression changes indicates that the TP53 network has an important role in an even higher proportion of tumors, translating into a clinically relevant patient subgroup with poor prognostic associations. There is currently no other curative therapy for malignant peripheral nerve sheath tumors than surgical resection [59], and we suggest that the biological knowledge of a prevalent "TP53 mutated phenotype" can be explored as a stratification parameter for novel treatments of this aggressive tumor type.

Acknowledgements We are grateful to Mette Eknæs at the Department of Molecular Oncology (Oslo University Hospital) for excellent technical assistance.

Funding This study was supported by the Norwegian Cancer Society (project number 6824048-2016 to AS and project number 72190-PR2006-0442 to RAL), the Southern and Eastern Norway Regional Health Authority (RAL), the foundation "Stiftelsen Kristian Gerhard Jebsen", and the Research Council of Norway.

\section{Compliance with ethical standards}

Conflict of interest The authors declare that they have no conflict of interest.

\section{References}

1. Kolberg M, Holand M, Agesen TH, et al. Survival meta-analyses for 1800 malignant peripheral nerve sheath tumor patients with and without neurofibromatosis type 1. Neuro Oncol. 2013;15:135-47.

2. Evans DG, Huson SM, Birch JM. Malignant peripheral nerve sheath tumours in inherited disease. Clin Sarcoma Res. 2012;2:17.

3. McCaughan JA, Holloway SM, Davidson R, et al. Further evidence of the increased risk for malignant peripheral nerve sheath tumour from a Scottish cohort of patients with neurofibromatosis type 1. J Med Genet. 2007;44:463-6.

4. Berner JM, Sorlie T, Mertens F, et al. Chromosome band 9p21 is frequently altered in malignant peripheral nerve sheath tumors: studies of CDKN2A and other genes of the pRB pathway. Genes Chromosomes Cancer. 1999;26:151-60.

5. Nielsen GP, Stemmer-Rachamimov AO, Ino Y, et al. Malignant transformation of neurofibromas in neurofibromatosis 1 is associated with $\mathrm{CDKN} 2 \mathrm{~A} / \mathrm{p} 16$ inactivation. Am J Pathol. 1999;155:1879-84.

6. Kourea HP, Orlow I, Scheithauer BW, et al. Deletions of the INK4A gene occur in malignant peripheral nerve sheath tumors but not in neurofibromas. Am J Pathol. 1999;155:1855-60.

7. Lothe RA, Karhu R, Mandahl N, et al. Gain of 17q24-qter detected by comparative genomic hybridization in malignant tumors from patients with von Recklinghausen's neurofibromatosis. Cancer Res. 1996;56:4778-81.
8. Mertens F, Dal Cin P, de Wever I, et al. Cytogenetic characterization of peripheral nerve sheath tumours: a report of the CHAMP study group. J Pathol. 2000;190:31-8.

9. Skotheim RI, Kallioniemi A, Bjerkhagen B, et al. TopoisomeraseII alpha is upregulated in malignant peripheral nerve sheath tumors and associated with clinical outcome. J Clin Oncol. 2003;21:4586-91.

10. Storlazzi CT, Brekke HR, Mandahl N, et al. Identification of a novel amplicon at distal $17 \mathrm{q}$ containing the BIRC5/SURVIVIN gene in malignant peripheral nerve sheath tumours. J Pathol. 2006;209:492-500.

11. Holtkamp N, Malzer E, Zietsch J, et al. EGFR and erbB2 in malignant peripheral nerve sheath tumors and implications for targeted therapy. Neuro Oncol. 2008;10:946-57.

12. Brekke HR, Ribeiro FR, Kolberg M, et al. Genomic changes in chromosomes 10,16 , and $\mathrm{X}$ in malignant peripheral nerve sheath tumors identify a high-risk patient group. J Clin Oncol. 2010;28:1573-82.

13. Lawrence MS, Stojanov P, Polak P, et al. Mutational heterogeneity in cancer and the search for new cancer-associated genes. Nature. 2013;499:214-8.

14. Lee $\mathrm{W}$, Teckie $\mathrm{S}$, Wiesner T, et al. PRC2 is recurrently inactivated through EED or SUZ12 loss in malignant peripheral nerve sheath tumors. Nat Genet. 2014;46:1227-32.

15. Zhang M, Wang Y, Jones S, et al. Somatic mutations of SUZ12 in malignant peripheral nerve sheath tumors. Nat Genet. 2014;46: $1170-2$.

16. Hirbe AC, Dahiya S, Miller CA, et al. Whole exome sequencing reveals the order of genetic changes during malignant transformation and metastasis in a single patient with NF1-plexiform neurofibroma. Clin Cancer Res. 2015;21:4201-11.

17. Bottillo I, Ahlquist T, Brekke H, et al. Germline and somatic NF1 mutations in sporadic and NF1-associated malignant peripheral nerve sheath tumours. J Pathol. 2009;217:693-701.

18. Verdijk RM, den Bakker MA, Dubbink HJ, et al. TP53 mutation analysis of malignant peripheral nerve sheath tumors. J Neuropathol Exp Neurol. 2010;69:16-26.

19. Dubbink HJ, Bakels H, Post E, et al. TERT promoter mutations and BRAF mutations are rare in sporadic, and TERT promoter mutations are absent in NF1-related malignant peripheral nerve sheath tumors. J Neurooncol. 2014;120:267-72.

20. Vogelstein B, Lane D, Levine AJ. Surfing the p53 network. Nature. 2000;408:307-10.

21. Nigro JM, Baker SJ, Preisinger AC, et al. Mutations in the p53 gene occur in diverse human tumour types. Nature. 1989;342: $705-8$.

22. Cichowski K, Shih TS, Schmitt E, et al. Mouse models of tumor development in neurofibromatosis type 1. Science. 1999;286: 2172-6.

23. Vogel KS, Klesse LJ, Velasco-Miguel S, et al. Mouse tumor model for neurofibromatosis type 1. Science. 1999;286:2176-9.

24. Zhang Y, Xiong Y, Yarbrough WG. ARF promotes MDM2 degradation and stabilizesp53: ARF-INK4a locus deletion impairs both the $\mathrm{Rb}$ and $\mathrm{p} 53$ tumor suppression pathways. Cell. 1998;92:725-34.

25. NCBI Pubmed. https://www.ncbi.nlm.nih.gov/pubmed/. Accessed Feb 132017

26. Fletcher DM, Bridge JA, Hogendoorn PC, et al. Malignant peripheral nerve sheath tumour. In: WHO classification of tumours of soft tissue and bone. 4th edn. Lyon, IRAC Press; 2013. p.187-89.

27. Karamchandani JR, Nielsen TO, van de Rijn M, et al. Sox10 and S100 in the diagnosis of soft-tissue neoplasms. Appl Immunohistochem Mol Morphol. 2012;20:445-50.

28. Miettinen MM, Antonescu CR, Fletcher CDM, et al. Histopathologic evaluation of atypical neurofibromatous tumors and their transformation into malignant peripheral nerve sheath tumor 
in patients with neurofibromatosis 1-a consensus overview. Hum Pathol. 2017;67:1-10.

29. Nonaka D, Chiriboga L, Rubin BP. Differential expression of S100 protein subtypes in malignant melanoma, and benign and malignant peripheral nerve sheath tumors. J Cutan Pathol. 2008;35:1014-9.

30. Schaefer IM, Fletcher CD, Hornick JL. Loss of H3K27 trimethylation distinguishes malignant peripheral nerve sheath tumors from histologic mimics. Mod Pathol. 2016;29:4-13.

31. Cleven AH, Sannaa GA, Briaire-de Bruijn I, et al. Loss of H3K27 tri-methylation is a diagnostic marker for malignant peripheral nerve sheath tumors and an indicator for an inferior survival. Mod Pathol. 2016;29:582-90.

32. Wang K, Li M, Hadley D, et al. PennCNV: an integrated hidden Markov model designed for high-resolution copy number variation detection in whole-genome SNP genotyping data. Genome Res. 2007;17:1665-74.

33. PennCNV Affy. Protocol for CNV detection in Affymetrix SNP arrays. http://www.openbioinformatics.org/penncnv/penncnv_ tutorial_affy_gw6.html. Accessed Mar 202017.

34. Sveen A, Loes IM, Alagaratnam S, et al. Intra-patient Intermetastatic Genetic Heterogeneity in Colorectal Cancer as a Key Determinant of Survival after Curative Liver Resection. PLoS Genet. 2016;12:e1006225.

35. McCarroll SA, Kuruvilla FG, Korn JM, et al. Integrated detection and population-genetic analysis of SNPs and copy number variation. Nat Genet. 2008;40:1166-74.

36. Van Loo P, Nordgard SH, Lingjaerde OC, et al. Allele-specific copy number analysis of tumors. Proc Natl Acad Sci USA. 2010;107:16910-5.

37. Beroukhim R, Getz G, Nghiemphu L, et al. Assessing the significance of chromosomal aberrations in cancer: methodology and application to glioma. Proc Natl Acad Sci USA. 2007;104: 20007-12.

38. Mermel CH, Schumacher SE, Hill B, et al. GISTIC2.0 facilitates sensitive and confident localization of the targets of focal somatic copy-number alteration in human cancers. Genome Biol. 2011;12: R41.

39. Nilsen G, Liestol K, Van Loo P, et al. Copynumber: Efficient algorithms for single- and multi-track copy number segmentation. BMC Genom. 2012;13:591.

40. Hanzelmann S, Castelo R, Guinney J. GSVA: gene set variation analysis for microarray and RNA-seq data. BMC Bioinforma. 2013;14:7.

41. IARC TP53 Database. Data file "somaticMutationsIARC TP53 Database, R18.txt". http://p53.iarc.fr/TP53SomaticMutations. aspx. Accessed Feb 12017.

42. ExAC Browser. Variant: 17: 7579472 G/C. http://exac.broa dinstitute.org/variant/17-7579472-G-C. Accessed Mar 272017.

43. Miller LD, Smeds J, George J, et al. An expression signature for p53 status in human breast cancer predicts mutation status, transcriptional effects, and patient survival. Proc Natl Acad Sci USA. $2005 ; 102: 13550-5$.
44. Lothe RA, Smith-Sorensen B, Hektoen M, et al. Biallelic inactivation of TP53 rarely contributes to the development of malignant peripheral nerve sheath tumors. Genes Chromosomes Cancer. 2001;30:202-6.

45. Rivlin N, Brosh R, Oren M, et al. Mutations in the p53 tumor suppressor gene: important milestones at the various steps of tumorigenesis. Genes Cancer. 2011;2:466-74.

46. Rahrmann EP, Moriarity BS, Otto GM, et al. Trp53 haploinsufficiency modifies EGFR-driven peripheral nerve sheath tumorigenesis. Am J Pathol. 2014;184:2082-98.

47. Brosius SN, Turk AN, Byer SJ, et al. Neuregulin-1 overexpression and Trp53 haploinsufficiency cooperatively promote de novo malignant peripheral nerve sheath tumor pathogenesis. Acta Neuropathol. 2014;127:573-91.

48. Pogribny IP, James SJ. Reduction of p53 gene expression in human primary hepatocellular carcinoma is associated with promoter region methylation without coding region mutation. Cancer Lett. 2002;176:169-74.

49. Almeida LO, Custodio AC, Pinto GR, et al. Polymorphisms and DNA methylation of gene TP53 associated with extra-axial brain tumors. Genet Mol Res: GMR. 2009;8:8-18.

50. Chmelarova M, Krepinska E, Spacek J, et al. Methylation in the p53 promoter in epithelial ovarian cancer. Clin Transl Oncol. 2013;15:160-3.

51. Amatya VJ, Naumann U, Weller M, et al. TP53 promoter methylation in human gliomas. Acta Neuropathol. 2005;110: 178-84.

52. Feber A, Wilson GA, Zhang L, et al. Comparative methylome analysis of benign and malignant peripheral nerve sheath tumors. Genome Res. 2011;21:515-24.

53. Rieske P, Bartkowiak JK, Szadowska AM, et al. A comparative study of P53/MDM2 genes alterations and P53/MDM2 proteins immunoreactivity in soft-tissue sarcomas. J Exp Clin Cancer Res. 1999;18:403-16.

54. Wallander ML, Tripp S, Layfield LJ. MDM2 amplification in malignant peripheral nerve sheath tumors correlates with p53 protein expression. Arch Pathol Lab Med. 2012;136:95-9.

55. Holtkamp N, Atallah I, Okuducu AF, et al. MMP-13 and p53 in the progression of malignant peripheral nerve sheath tumors. Neoplasia. 2007;9:671-7.

56. Kolberg M, Bruun J, Murumagi A, et al. Drug sensitivity and resistance testing identifies PLK1 inhibitors and gemcitabine as potent drugs for malignant peripheral nerve sheath tumors. Mol Oncol. 2017;11:1156-71.

57. Xie Y, Wang A, Lin J, et al. Mps1/TTK: a novel target and biomarker for cancer. J Drug Target. 2017;25:112-8.

58. Murahashi M, Hijikata Y, Yamada K, et al. Phase I clinical trial of a five-peptide cancer vaccine combined with cyclophosphamide in advanced solid tumors. Clin Immunol. 2016;166-7:48-58.

59. Bradford D, Kim A. Current treatment options for malignant peripheral nerve sheath tumors. Curr Treat Options Oncol. 2015; $16: 328$. 